\title{
COMPLEXIDADE DA FARMACOTERAPIA PÓS-TRANSPLANTE RENAL: INFLUÊNCIA NA ADESÃO AO TRATAMENTO
}

Complexity of post-renal transplant pharmacotherapy: influence treatment adherence

La complejidad de la farmacoterapia post-trasplante renal: influencia en la adhesión al tratamiento

Ana Carolina de Souza e Silva1', Bruna Cristina Cardoso Martins², Liana Silveira Adriano1,

Marta Maria de França Fonteles ${ }^{1,2}$, Pedro Henrique Veras Reis ${ }^{3}$, Elana Figueiredo Chaves ${ }^{1 *}$,

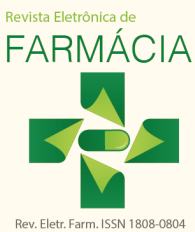

1 Faculdade de Farmácia, Universidade Federal do Ceará

2 Programa de Pós-Graduação em Ciências Farmacêuticas,

Faculdade de Farmácia da Universidade Federal do Ceará

${ }^{3}$ Universidade Federal de Sergipe

*E-mail: elana_figueiredo@yahoo.com.br

\section{Resumo}

Objetivo: Analisar a influência da complexidade da farmacoterapia (CFT) na adesão ao tratamento imunossupressor.

Métodos: Trata-se de um estudo analítico, observacional e transversal, baseado nos registros do atendimento farmacêutico de pacientes transplantados renais de um hospital universitário (Fortaleza/Ceará). A CFT foi avaliada em consulta farmacêutica no período de janeiro a julho/2014, utilizando-se o Índice de Complexidade da Farmacoterapia (ICFT). A análise da adesão foi realizada através do nível sérico dos inibidores da calcineurina e imTor.

Resultados: Analisou-se o acompanhamento de 36 pacientes: sendo 52,78\% ( $n=19)$ homens; $27,80 \%$ ( $n=1$ 0) entre 41 e 50 anos; $41,70 \%$ ( $n=15)$ com ensino fundamental incompleto e 72,22\% ( $n=26)$ possuíam cuidador. A média de medicamentos foi nove e de pontos no ICFT foi 50,94. O ICFT máximo foi registrado na faixa de 31-60 dias pós-transplante. Analisando o nível sérico dos imunossupressores, observou-se prevalência de pacientes classificados como "não aderentes" maior no período menor de 60 dias pós-transplante.

Conclusão: Concluiu-se que no pós-transplante renal a adesão dos pacientes ao tratamento imunossupressor aumenta com a diminuição da CFT, sendo necessária uma maior e melhor orientação dos pacientes no inicio do pós-transplante.

Palavras-chave: Transplante de Rim. Adesão do Paciente. Imunossupressores. Atenção Farmacêutica.

\section{Abstract}

Objective: This study aimed to analyze the influence of pharmacotherapeutical complexity on immunosuppressive treatment adherence.

Methods: Analytical, observational and cross-sectional study was performed, based on records of renal transplant patient pharmaceutical service from a university hospital (Fortaleza/Ceara). Complexity of pharmacotherapy was evaluated in the first patient-pharmacist appointment during January-July/2014. The Medication Regimen Complexity Index (MRCI) was employed, which included pharmaceutical form, posology and instructions through scores classification. Adherence analysis was evaluated by measurement of serum levels of inhibitors of calcineurin and mTOR.

Results: Monitoring of 36 patients was performed, being $52.78 \%(n=19)$ men; $27.80 \%(n=10)$ between 41 and 50 years old; $41.70 \%(n=15)$ with incomplete primary education; and $72.22 \%(n=26)$ had a caregiver. The mean of drugs was nine and of $\mathrm{MRCl}$ points was 50.94. The maximum $\mathrm{MRCI}$ was observed between 31-60 days after transplant. Regarding serum levels of 
immunosuppressives, a greater prevalence of patients classified as "non-adherent" was observed during post-transplant period less than 60 days.

Conclusion: During post-renal transplant period, adhesion of patients to immunosuppressive treatment increases as the complexity of pharmacotherapy decreases, indicating the need of a greater and better orientation of the patients during the post-transplant initial period.

Keywords: Kidney Transplantation. Patient compliance. Immunosuppressive agents. Pharmaceutical care.

\section{Resumen}

Objetivo: Analizar la influencia de la complejidad de la farmacoterapia (CFT) en la adhesión al tratamiento inmunosupresor.

Métodos: Se trata de un estudio analítico, observacional y transversal basado en los registros de atención farmacéutica de pacientes con trasplantes renales de un hospital universitario (Fortaleza/Ceará). La CFT fue evaluada en el periodo de enero a julio de 2014, utilizando el Índice de Complejidad de la Farmacoterapia (ICFT). El análisis de la adhesión se realizó utilizando los niveles séricos de los inhibidores de la calcineurina e imTor.

Resultados: Se analizó el acompañamiento de 36 pacientes: siendo $52.78 \%(n=19)$ hombres; $27,80 \%(n=10)$ entre 41 y 50 años; $41,70 \%(n=15)$ con educación primaria incompleta y el $72,22 \%(n=26)$ poseía un cuidador. El promedio de medicamentos fue de nueve y el promedio de puntos en el ICFT fue de 50,94 puntos. El ICFT máximo se registró en la faja de 31-60 días post-trasplante. En el análisis de los niveles séricos de inmunosupresores, se observó que prevalecieron pacientes clasificados "no adheridos" en el periodo menor de 60 días después del trasplante.

Conclusión: Se concluyó que en el post trasplante renal la adhesión al tratamiento inmunosupresor aumenta con la disminución de la CFT, lo que requiere una mayor y mejor orientación de los pacientes durante el inicio del post trasplante.

Palabras clave: Trasplante renal. Adhesión del paciente. Inmunosupresores. Atención farmacéutica.

\section{INTRODUÇÃO}

O transplante renal é uma das modalidades de tratamento e reabilitação mais recomendadas para pacientes com insuficiência renal crônica, por oferecer melhor qualidade de vida ao paciente, uma possível redução do risco de mortalidade dependendo das características do paciente - e custo financeiro menor do que a diálise. No entanto, a escolha por esse tipo de tratamento deve levar em conta as características individuais dos pacientes, ou seja, fatores demográficos e de comorbidade, pois cada modalidade de tratamento possui vantagens e desvantagens ${ }^{(1)}$.

No Brasil, os transplantes iniciaram na década de 60 , quando houve, em 1964, o primeiro transplante de rim, no estado do Rio de Janeiro(2). O estado do Ceará tem destaque no país, realizando, em 2013, 329 transplantes renais, sendo 112 realizados no Hospital Universitário Walter Cantídio ${ }^{(3-4)}$. Em todo o Brasil, o Hospital Universitário Walter Cantídio ocupa o quarto lugar em número de transplantes renais, atrás apenas de São Paulo, Santa Catarina e Rio Grande do Sul e foi o pioneiro nessa área, realizando, em 1977, o primeiro transplante de rim do Ceará e da região Nordeste ${ }^{(5-6)}$.

As complicações decorrentes do transplante podem ser minimizadas à medida que o paciente segue todas as recomendações médicas, especialmente as relacionadas com a farmacoterapia. O tratamento medicamentoso requer o uso de imunossupressores (corticosteróides, inibidores da calcineurina, antimetabólitos e/ou inibidores da mTOR), fazendo-se 
necessária uma orientação prévia, para que esses medicamentos sejam utilizados adequadamente e garantam a efetividade do tratamento ${ }^{(7-8)}$.

A farmacoterapia imunossupressora é extremamente importante na assistência ao paciente pós-transplante, que deve fazer uso crônico de imunossupressores, a fim de evitar episódios de rejeição e consequente perda do enxerto ${ }^{(7-8)}$. Entretanto, a complexidade da terapia pode ser um interferente na adesão ao tratamento, devendo ser avaliada a fim de identificar fatores determinantes, evitar complicações que podem levar à perda do enxerto e proporcionar aos pacientes um tratamento mais seguro(9). $^{(9)}$.

A complexidade da farmacoterapia é baseada nas múltiplas características do regime prescrito, incluindo o número de diferentes medicamentos no esquema, o número de doses diárias, o número de unidades de dosagem por dose e as relações da dose com a alimentação. Para a avaliação dessa complexidade, George et al. propôs um instrumento que permite quantificar a complexidade da farmacoterapia, o Medication Regimen Complexity Index $(\mathrm{MRCl})$, já validado na versão para a língua portuguesa (Índice de Complexidade da Farmacoterapia - ICFT) ${ }^{(9-12)}$.

A polifarmácia é frequente em pacientes transplantados que, além dos fármacos imunossupressores, utilizam também medicamentos profiláticos, pois ficam mais propensos a infecções por vírus, bactérias e fungos. Em alguns casos, há ainda tratamento para doenças crônicas, como hipertensão e diabetes. Portanto, o risco de ocorrência de reações adversas, interações medicamentosas e de dificuldades na utilização dos medicamentos aumenta consideravelmente, sendo necessário um acompanhamento mais intensivo desses pacientes ${ }^{(8,13-14)}$.

É nesse contexto que os farmacêuticos são inseridos nas equipes multiprofissionais de assistência ao paciente transplantado, pois podem contribuir para a redução de problemas relacionados a medicamentos e garantir um acompanhamento farmacoterapêutico pós-transplante adequado, contribuindo para a segurança do paciente, redução da mortalidade e da hospitalização associadas a medicamentos ${ }^{(8,13,15)}$. O presente trabalho buscou analisar a complexidade da farmacoterapia de pacientes transplantados renais atendidos no serviço de atenção farmacêutica do ambulatório de transplante renal e sua relação com a adesão ao tratamento.

\section{METODOLOGIA}

Trata-se de um estudo observacional, descritivo e transversal, fundamentado na avaliação do registro de atendimento farmacêutico de pacientes acompanhados pelo Serviço de Atenção Farmacêutica (SATENFAR), no ambulatório de transplante renal no Hospital Universitário Walter Cantídio (Fortaleza/Ceará), no período de janeiro a julho de 2014. Os dados foram coletados em uma Ficha de Acompanhamento Farmacoterapêutica (FAFT) própria, permitindo coleta de dados sociodemográficos, de escolaridade, comorbidades, cuidador do paciente, sobre o tratamento farmacológico instituído e níveis séricos dos medicamentos imunossupressores. Foram excluídos da pesquisa: 1) as FAFT que apresentaram falta de informações ou dados incompletos; 2) pacientes que foram transferidos para seu estado de origem, sofreram perda do enxerto e/ou retornam para a fila de transplante.

A análise da adesão foi realizada através do nível sérico dos inibidores da calcineurina e inibidores da mTor como método direto de avaliação de adesão. O nível sérico dos imunossupressores foi classificado de acordo com o protocolo do Serviço de Transplante Renal do hospital (2012)(6). As informações sobre a complexidade da terapia farmacológica de cada paciente foram coletadas nos registros da primeira consulta realizada pelo paciente no período analisado, sendo avaliados através do instrumento chamado Índice de Complexidade da Farmacoterapia (ICFT), na versão portuguesa validada(9). O ICFT é dividido em três seções: A, B e C. A seção " $A$ " corresponde às informações relacionadas às formas de dosagens; a seção "B", faz referência 
às frequências de doses; e a seção " $\mathrm{C}$ " corresponde às informações adicionais, como uso concomitante com alimentos, entre outras. A pontuação de cada seção foi obtida a partir da análise da farmacoterapia do paciente e o índice de complexidade foi obtido pela soma dos pontos (escores) das três seções.

Através dos dados coletados, foram calculadas as porcentagens de cada variável e analisadas no programa estatístico EPI-INFO v. 3.5.1. para tabulação dos dados. A avaliação da prevalência de não adesão dos pacientes identificados como mais complexos, de acordo com ICFT, foi realizada como medida de associação o Risco de Prevalência (RP). Alguns resultados foram expressos em média e desvio padrão e apresentados na forma de gráficos e/ou tabela. Realizou-se análise estatística no programa SigmaPlot v. 13, em que o teste " $t$ " foi aplicado sendo considerado significante $p<0,05$. Este estudo foi elaborado de acordo com as normas do Conselho Nacional de Saúde (Resolução CNS 466, Dezembro 2012) e aprovado pelo Comitê de Ética e Pesquisa da Universidade Federal do Ceará (CAAE 05925513.2.1001.5054).

\section{RESULTADOS}

No período analisado foram atendidos 40 pacientes transplantados no SATENFAR. Foram excluídos os registros de 4 pacientes por não se enquadrarem aos critérios de inclusão. Desta forma, a população analisada englobou a FAFT de 36 pacientes (Tabela 1).

Quanto ao perfil sociodemográfico dos pacientes, observa-se que $52,78 \%(n=19)$ eram do sexo masculino, com idade variando de 21 a 80 anos, sendo que $27,80 \%(n=10)$ dos pacientes estavam na faixa etária de 41 a 50 anos e $41,7 \%(n=15)$ com ensino fundamental incompleto (Tabela 1).

A maior parte dos pacientes era proveniente de outras localidades $(n=21 ; 58,30 \%)$. A presença de cuidador foi expressiva na amostra analisada $(72,22 \%, \mathrm{n}=26)$. Na amostra analisada, a maior parte desses cuidadores tinha ensino fundamental incompleto $(27,78 \%, n=10)$ ou ensino médio completo $(27,78 \%, n=10)$, sendo a maioria esposas $(25 \%$, $n=9)$ e filhas $(25 \%$, $n=9)$. Em relação às características clínicas dos pacientes estudados, observou-se que $86,11 \%(n=31)$ dos pacientes possuíam hipertensão, enquanto $22,22 \%$ (n=8) possuíam diabetes (Tabela 1).

Tabela 1. Distribuição das frequências segundo as características sociodemográficas de pacientes atendidos pelo farmacêutico no ambulatório de transplante renal do HUWC em Fortaleza, Ceará ( $N=36)$, no período de janeiro a julho de 2014

\begin{tabular}{|l|r|r|l|l|l|l|}
\hline Variáveis & N & $\%$ & Variáveis & N & $\%$ \\
\hline \multicolumn{7}{|c|}{ PACIENTE } \\
\hline Sexo & 19 & 52,78 & Fortaleza & 15 & 41,7 \\
\hline Masculino & 17 & 47,22 & Outras localidades & 21 \\
\hline Feminino & & & Escolaridade do Paciente & 58,3 \\
\hline Faixa etária & 4 & 11,1 & Analfabeto & 5 & 13,9 \\
\hline $21-30$ anos & 9 & 25 & Fundamental Completo & 2 & 5,6 \\
\hline $31-40$ anos & & & & \\
\hline
\end{tabular}




\begin{tabular}{|c|c|c|c|c|c|}
\hline $41-50$ anos & 10 & 27,8 & Fundamental Incompleto & 15 & 41,7 \\
\hline $51-60$ anos & 7 & 19,4 & Médio Completo & 12 & 33,3 \\
\hline $61-70$ anos & 4 & 11,1 & Não Consta & 1 & 2,8 \\
\hline $71-80$ anos & 2 & 5,6 & Superior Completo & 1 & 2,8 \\
\hline \multicolumn{3}{|l|}{ Diabetes } & \multicolumn{3}{|l|}{ Hipertensão } \\
\hline Não & 25 & 69,44 & $\operatorname{sim}$ & 31 & 86,11 \\
\hline Sim & 8 & 22,22 & Não & 3 & 8,33 \\
\hline Não houve resposta & 3 & 8,33 & Não houve resposta & 2 & 5,56 \\
\hline \multicolumn{6}{|c|}{ CUIDADOR } \\
\hline \multicolumn{3}{|l|}{ Parentesco com o paciente } & \multicolumn{3}{|l|}{ Grau de escolaridade } \\
\hline Não possui cuidador & 10 & 27,78 & Fundamental Completo & 1 & 2,78 \\
\hline Esposa & 9 & 25 & Fundamental Incompleto & 10 & 27,78 \\
\hline Filha & 9 & 25 & Médio Completo & 10 & 27,78 \\
\hline Filho & 2 & 5,56 & Médio Incompleto & 2 & 5,56 \\
\hline Esposo & 2 & 5,56 & Superior Completo & 2 & 5,56 \\
\hline Mãe & 2 & 5,56 & Superior Incompleto & 1 & 2,78 \\
\hline Outros graus de parentesco & 2 & 5,56 & Não possui cuidador & 10 & 27,78 \\
\hline
\end{tabular}

Fonte: Elaborada pelos autores, 2016.

Na análise do perfil farmacoterapêutico dos medicamentos utilizados pelos pacientes a média de consumo foi de nove medicamentos por paciente, havendo uma prevalência de medicamentos do grupo dos anti-infectantes gerais para uso sistêmico (Grupo J) (n=76; 22,96\%), na primeira consulta (Tabela 2).

Tabela 2. Medicamentos utilizados pelos pacientes atendidos pelo farmacêutico no ambulatório de transplante renal do HUWC - primeira e última consulta. Janeiro - Julho/2014, Fortaleza, Ceará

\section{GRUPOS ANATÔMICOS PRINCIPAIS DO SISTEMA ATC*}

Aparelho digestivo e metabolismo

Sangue e órgãos hematopoiéticos

\begin{tabular}{|c|r|}
\hline CÓDIGO ATC* & $\mathbf{N}(\%)$ \\
\hline A & $63(19,03)$ \\
\hline B & $22(6,64)$ \\
\hline
\end{tabular}




\begin{tabular}{|l|c|r|}
\hline Sistema Cardiovascular & C & 58(17,52) \\
\hline Dermatológicos & $\mathrm{G}$ & $10(3,02)$ \\
\hline Sistema geniturinário e hormônios sexuais & $\mathrm{H}$ & $21(6,34)$ \\
\hline Hormônios de uso sistêmico, excluindo hormônios sexuais & $\mathrm{J}$ & $76(22,96)$ \\
\hline Anti-infectantes gerais para uso sistêmico & $\mathrm{L}$ & $72(21,75)$ \\
\hline Antineoplásicos e agentes moduladores do sistema imunológico & $\mathrm{M}$ & $2(0,60)$ \\
\hline Sistema Músculo esquelético & $\mathrm{N}$ & $5(1,51)$ \\
\hline Sistema nervoso central & $\mathrm{R}$ & $1(0,30)$ \\
\hline Sistema respiratório & - & $331(100)$ \\
\hline Total & & \\
\hline
\end{tabular}

*Anatomical Therapeutic Chemical (ATC)

Fonte: Elaborada pelos autores, 2015.

A complexidade terapêutica avaliada através da aplicação do ICFT apresentou, para a primeira consulta pós-transplante com o farmacêutico, uma média total de 50,94 pontos $( \pm 12,37)$ (tabela 3).

A seção do ICFT que mais contribuiu para o aumento da complexidade terapêutica foi a " $\mathrm{C}$ " - instruções especiais, com uma pontuação média de 33,44. As instruções especiais mais prevalentes foram: múltiplas unidades ao mesmo tempo; tomar/ usar em horário específico; relação com o alimento e tomar/usar conforme indicado (tabela 3).

Tabela 3. Valores médios, máximos e mínimos do Índice de Complexidade Terapêutico e das suas seções de pacientes atendidos pelo farmacêutico no ambulatório de transplante renal do HUWC. Janeiro - Julho/2014, Fortaleza, Ceará

\begin{tabular}{|l|l|l|l|}
\hline \multirow{2}{*}{ Índice de Complexidade da Prescrição } & \multicolumn{2}{c|}{$1^{\text {a Consulta }}$} & Mínimo \\
\cline { 2 - 4 } & \multicolumn{1}{|c|}{ Média dos pontos } & 10 & 1 \\
\hline Seção A & $3( \pm 2,3)$ & 25 & 8,5 \\
\hline Seção B & $14,5( \pm 4,0)$ & 56 & 21 \\
\hline Seção C & $33,44( \pm 7,4)$ & 81,5 & 31,5 \\
\hline Total & $50,94( \pm 12,3)$ & & \multirow{2}{*}{} \\
\hline
\end{tabular}

Fonte: Elaborada pelos autores, 2015.

É importante salientar ainda que, dentro da seção "A", a forma farmacêutica mais utilizada pelos pacientes transplantados da amostra estudada foi comprimido/cápsula. Na seção "B", a frequência mais usual foi uma vez por dia e duas vezes por dia 
e na seção "C", as instruções especiais mais prevalentes foram: múltiplas unidades ao mesmo tempo; tomar/usar em horário específico; relação com o alimento e tomar/usar conforme indicado.

Neste estudo, a maior pontuação obtida foi 79,5 pontos referentes à utilização de 13 medicamentos e valor mínimo de 24,5 pontos associado à administração de 4 medicamentos diariamente, o que corrobora com o fato de o número de medicamentos não ser o único fator que interfere na complexidade ${ }^{(9)}$. Além disso, observaram-se valores diferentes de complexidade para esquemas terapêuticos com o mesmo número de fármacos e, também, valores iguais de complexidade para esquemas com número de fármacos diferentes.

Os ICFT, na análise da primeira consulta do paciente, com maiores pontuações ficaram localizados nas faixas de 15-30 dias e 31-60 dias, sendo o máximo (81,5 pontos) registrado em um paciente pertencente à faixa de 31-60 dias no tempo póstransplante (Tabela 4).

Tabela 4. Tempo pós-transplante versus ICFT de pacientes atendidos pelo farmacêutico no ambulatório de transplante renal do HUWC Janeiro- Julho/2014, Fortaleza, Ceará

\begin{tabular}{|c|c|c|c|c|c|c|}
\hline $1^{\text {a }}$ consulta & \multicolumn{6}{|c|}{ ICFT } \\
\hline $\begin{array}{l}\text { Tempo pós- trans- } \\
\text { plante }\end{array}$ & $\begin{array}{c}\text { 31-40pts } \\
\%(n)\end{array}$ & $\begin{array}{c}\text { 41-50pts } \\
\%(n)\end{array}$ & $\begin{array}{c}\text { 51-60pts } \\
\%(n)\end{array}$ & $\begin{array}{c}\text { 61-70pts } \\
\%(n)\end{array}$ & $\begin{array}{l}>71 p t s \\
\%(n)\end{array}$ & $\begin{array}{l}\text { TOTAL } \\
\%(n)\end{array}$ \\
\hline 15-30 dias & $9,1(1)$ & $27,3(3)$ & $27,3(3)$ & $18,2(2)$ & $18,2(2)$ & $100(11)$ \\
\hline $31-60$ dias & $7,7(1)$ & $38,5(5)$ & $38,5(5)$ & $7,7(1)$ & $7,7(1)$ & $100(13)$ \\
\hline $61-180$ dias & $40(2)$ & $40(2)$ & $20(1)$ & 0 & 0 & $100(5)$ \\
\hline 6 meses -1 ano & $60(3)$ & $40(2)$ & 0 & 0 & 0 & $100(5)$ \\
\hline$>1$ ano & 0 & $50(1)$ & $50(1)$ & 0 & 0 & $100(2)$ \\
\hline TOTAL & $19,4(7)$ & $36,1(13)$ & $27,8(10)$ & $8,3(3)$ & $8,3(3)$ & $100(36)$ \\
\hline
\end{tabular}

Fonte: Elaborada pelos autores, 2015.

Em relação à terapia imunossupressora, observou-se que o esquema mais prevalente foi a combinação dos medicamentos tacrolimo, micofenolato de sódio e prednisona ( $n=17 ; 47,2 \%$ ) (esquema tríplice preconizado no protocolo do serviço), seguido pela associação de tacrolimo e micofenolato de sódio $(n=13 ; 36,1 \%)$, e tacrolimo e everolimo ( $n=2 ; 5,5 \%)$. Houve um paciente em cada associação de micofenolato de sódio, everolimo e prednisona (2,8\%); tacrolimo, everolimo e prednisona (2,8\%); tacrolimo, everolimo e micofenolato de sódio (2,8\%); tacrolimo e prednisona (2,8\%).

Em relação ao nível sérico dos imunossupressores pertencentes à classe dos inibidores da calcineurina ou inibidores da mTor associado ao período pós-transplante em que o paciente se encontrava, observou-se que o grupo pertencente, na primeira consulta ao período de 15-30 dias teve uma maior frequência de pacientes classificados como não aderentes ao tratamento $(\mathrm{N}=7 ; 63,6 \%)$. Avaliando-se os outros períodos em que o paciente se encontrava quando realizou a primeira consulta com o farmacêutico, percebe-se que quanto mais distante do transplante menor é a taxa de não adesão (Tabela 5). 
Tabela 5. Avaliação direta da adesão ao tratamento em relação ao período pós-transplante de pacientes atendidos pelo farmacêutico no ambulatório de transplante renal do HUWC. Janeiro- Julho/2014, Fortaleza, Ceará

\begin{tabular}{|l|l|l|l|}
\hline \multirow{2}{*}{ Tempo pós- transplante } & \multicolumn{1}{|c|}{$\mathbf{1}^{\text {a } \text { CONSULTA }}$} & \multicolumn{1}{c|}{ Não aderente } & Total \\
\cline { 2 - 4 } & \multicolumn{1}{|c|}{ Aderente } & $63,6 \%(\mathrm{~N}=7)$ & $\mathrm{N}=11$ \\
\hline $\mathbf{1 5 - 3 0}$ dias & $36,3 \%(\mathrm{~N}=4)$ & $46,1 \%(\mathrm{~N}=6)$ & $\mathrm{N}=13$ \\
\hline $\mathbf{3 1 - 6 0}$ dias & $53,8 \%(\mathrm{~N}=7)$ & $14,3 \%(\mathrm{~N}=1)$ & $\mathrm{N}=7$ \\
\hline $\mathbf{6 1 - 1 8 0}$ dias & $85,7 \%(\mathrm{~N}=6)$ & $33,4 \%(\mathrm{~N}=1)$ & $\mathrm{N}=3$ \\
\hline $\mathbf{6}$ meses-1 ano & $66,6 \%(\mathrm{~N}=2)$ & $100 \%(\mathrm{~N}=2)$ & $\mathrm{N}=2$ \\
\hline $\mathbf{1}$ ano & $0 \%$ & $\mathrm{~N}=17$ & \\
\hline TOTAL & $\mathrm{N}=19$ & & \\
\hline
\end{tabular}

Teste t: $\mathrm{p}=0,831$-Primeira consulta.

A análise do perfil farmacoterapêutico associado ao período em que o paciente se encontrava durante a primeira consulta mostrou que a razão de prevalência de não adesão no período pós-transplante renal <60 dias é 1,6 vezes maior do que no período $>60$ dias.

\section{DISCUSSÃO}

A faixa etária dos pacientes acompanhados foi elevada, sendo associado a um maior número de pacientes com baixa escolaridade, o que pode contribuir para uma menor compreensão da farmacoterapia instituída, configurando um perfil semelhante ao encontrado por Martins et al., em Fortaleza, e Cunha et al., no Rio de Janeiro ${ }^{(1,14)}$. Já foi descrito na literatura que a idade e o grau de escolaridade podem estar relacionados com o grau de adesão do paciente à farmacoterapia ${ }^{(16)}$. A maioria dos pacientes apresentara como doença de base causadora da insuficiência renal o diabetes ou a hipertensão. Essas são as principais patologias causadoras da insuficiência renal crônica, bem como são complicações decorrentes do uso de corticóide e imunossupressores ${ }^{(1)}$.

A polimedicação no pós-transplante é um fato comum ${ }^{(8)}$. Embora trabalhos que mensurem e confirmem a alta complexidade da farmacoterapêutica pós-transplante seja limitada, a complexidade da farmacoterapia é suscetível de ser elevada em razão de esquemas farmacoterapêuticos e as frequentes mudanças de dosagem ${ }^{(24)}$. Torna-se essencial a presença de um cuidador com um nível de escolaridade mais avançado; normalmente quem assume esse papel são os próprios membros da família(14,17). Segundo Melo, Rodrigues, Schmidt (2009), o cuidador é a pessoa responsável por auxiliar o paciente em suas atividades e rotinas; este tem papel fundamental na provisão de cuidados, pois é quem assume e garante a manutenção da assistência necessária ao paciente em seu domicílio(17).

O número de medicamentos utilizados pelo paciente tem grande influência na complexidade, mas não pode ser considerado único fator ${ }^{(22)}$. No estudo de Borges et al. (21), a seção " $\mathrm{C}$ " foi também a que mais contribuiu para o aumento da complexidade 
terapêutica assim como encontrado nesse trabalho. No estudo de complexidade da terapêutica geriátrica realizado por Borges et al. (2011), a complexidade avaliada através da aplicação do ICFT apresentou uma média de 30,56 pontos, atingindo um máximo de 67 pontos e um mínimo de 13,50 pontos ${ }^{(21)}$. Esse dado foi diferente do apresentando no estudo com paciente transplantado renal que atingiu uma pontuação maior do que o paciente geriátrico.

A farmacoterapia imunossupressora é um aspecto extremamente importante no atendimento ao paciente póstransplantado, pois, assim, pode-se evitar a rejeição de órgãos desencadeada por fatores imunológicos, sendo a principal condição limitadora para o sucesso do transplante ${ }^{(8,23)}$. Embora seja necessária, a monitorização contínua do paciente transplantado, o período pós-transplante inicial é especialmente crítico, sendo a duração desse período o primeiro ano ${ }^{(8)}$.

Os níveis fora da recomendação do protocolo são indicativos de não adesão, conforme foi considerado nesse estudo. Entretanto, devido a outros fatores que podem influenciar no nível do imunossupressor, essa relação é apenas sugestiva. É importante salientar que, na primeira consulta, a maioria dos pacientes $(n=24)$ tinha entre 15-60 dias de transplante, período de adaptação e com prescrições mais complexas e, na segunda consulta, a maioria $(n=30)$ tinha entre 60 dias e um ano e possivelmente já compreendiam melhor o tratamento. Em relação à estatística, o número pequeno de pacientes pode ter levado os dados a não serem estatisticamente significantes.

O tratamento estabelecido no pós-transplante renal é extremamente complexo. A adesão ao tratamento é um fator importante para o sucesso do transplante e, a fim de evitar a rejeição aguda e perda do enxerto dos pacientes transplantados, os pacientes necessitam de um atendimento específico com orientações adequadas ${ }^{(18-20)}$. A utilização de muitos medicamentos aumenta o risco de ocorrência de reações adversas e interações medicamentosas, fatores que podem resultar em abandono do tratamento, contribuindo ainda mais para a não adesão do transplantado ao tratamento ${ }^{(13-14)}$.

\section{CONCLUSÃO}

Os pacientes transplantados renais atendidos no serviço de atenção farmacêutica tiveram uma maior adesão ao tratamento imunossupressor com a diminuição da CFT. Além disso, foi evidenciada uma maior complexidade da farmacoterapia no período menor que sessenta dias, associada a uma menor adesão. Os fatores que mais influenciaram nessa complexidade e possível diminuição da adesão foram as instruções especiais (múltiplas unidades ao mesmo tempo; tomar/usar em horário específico; relação com o alimento e tomar/usar conforme indicado) e o número de medicamentos a serem administrados diariamente que diminuíram ao logo do tempo do pós-transplante renal.

\section{REFERÊNCIAS}

1. Cunha CB, Leon ACP, Schramm JMA, Carvalho MS, Souza Jr PRB, Chain R. Tempo até o transplante e sobrevida em pacientes com insuficiência renal crônica no Estado do Rio de Janeiro, Brasil, 1998-2002. Cad. Saúde Pública. 2007;4(23):805-812. DOI: 10.1590/S0102-311X2007000400008.

2. Borges MC, Batista MOR, Rodrigues AMM, Carvalho OMC. Transplante de fígado no Ceará: Caracterização da população atendida em 2007. Revista de Pesquisa: Cuidado É Fundamental Online. 2010; 2 Suppl:5-7.

3. Associação Brasileira de Transplante de Órgãos. [homepage na internet]. Dimensionamento dos transplantes no Brasil e em cada estado (2006-2013). [cited 2014 aug 11]. Author's manuscript available at: http://www.abto.org.br/abtov03/default. aspx?mn=557\&c=1062\&s=0\&friendly=rbt-\%BB-2013---ano-xix-n4 
4. Hospital Universitário Walter Cantídio [homepage na internet]. Hospital Walter Cantídio é destaque em transplantes de fígado na América Latina. [cited 2014 aug 11]. Available from: www.huwc.ufc.br

5. Hospital Universitário Walter Cantídio [homepage na internet]. Primeiro transplante renal no HUWC completa 36 anos. [cited 2014 aug 11]. Available from: www.huwc.ufc.br

6. Hospital Universitário Walter Cantídio [homepage na internet]. Transplante Renal. [cited 2015 dic 12]. Available from: www. huwc.ufc.br

7. Lee MA. Transplantation: drug aspects of immunosuppression. The Pharmaceutical Journal. 2003; 10:201-7.

8. Stemer G, Lemmens-Gruber R. Clinical pharmacy services and solid organ transplantation: a literature review. Springer Science+Business Media B.V. 2009. Pharm. World Sci. 2010;32:7-18. DOI: 10.1007/s11096-009-9351-7.

9. Melchiors AC, Correr CJ, Fernandez-Llimos F. Translation and validation into Portuguese language of the medication regimen complexity index. Arq. Bras. Cardiol. 2007; 89(4):210-218. DOI: 10.1590/S0066-782X2007001600001.

10. Svarstad BI, Bultman DC. The patient: behavioral determinants. The Science and Practice of Pharmacy. 2000; 20:1948-56.

11. Stone VE, Hogan JW, Schuman P, Rompalo AM, Howard AA, Korkontzelou C, et al. Antiretroviral regimen complexity, self-reported adherence, and HIV patients' understanding of their regimens: survey of women in the HER study. J. Acquir. Immune Defic. Syndr. 2001; 28(2):124-31.

12. George J, Phun YT, Bailey MJ, Kong DC, Stewart K. Development and validation of the medication regimen complexity index. Ann. Pharmacother. 2004;38(9):1369-76. DOI: 10.1345/aph.1D479.

13. Wang HY, Chan ALF, Chen MT, Liao CH, Tian YF. Effects of pharmaceutical care intervention by clinical pharmacists in renal transplant clinics. Transplant. Proc. 2008; 40(7):2319-23. DOI: 10.1016/j.transproceed.2008.06.050.

14. Martins BCC, Souza TR, Luna AMPT, Fonteles MMF, Firmino FYM, Fernandes PFCBC, et al. Pharmaceutical care in transplant patients in a university hospital: pharmaceutical interventions. RBCF. 2013; 49(4):659-668.

15. Viktil KK, Blix HS. The Impact of Clinical Pharmacists on Drug-Related Problems and Clinical Outcomes. Basic Clin. Pharmacol. Toxicol. 2008;102(3):275-280. DOI: 10.1111/j.1742-7843.2007.00206.x.

16. Lima TM, Meiners MMMA, Soler O. Treatment adherence profile of hypertension patients from the Municipal Health Unit of Fátima, City of Belém, Pará, Amazônia, Brazil. Rev. Pan-Amaz. Saúde. 2010; 1(2):113-120.

17. Melo TM, Rodrigues IG, Shimidt DRC. Caracterização dos cuidadores de pacientes em cuidados paliativos no domicílio. Revista Brasileira de Cancerologia. 2009; 4(55):365-374.

18. Nevins TE, Kruse L, Skeans MA, Thomas W. The natural history of azathioprine compliance after renal transplantation. Kidney Int. 2001; 60:1565-1570. DOI:10.1046/j.1523-1755.2001.00961.x.

19. Butler JA, Peveler RC, Roderick P, Smith PW, Horne R, Mason JC. Modifiable risk factors for non-adherence to immunosuppressants in renal transplant recipients: a cross-sectional study. Nephrol. Dial. Transplant. 2004; 19(12):3144-49. DOI: 10.1093/ndt/gfh505.

20. Vlaminck H, Maes B, Evers G, Verbeke G, Lerut E, Van Damme B, et al. Prospective study on late consequences of subclinical non-compliance with immunosuppressive therapy in renal transplant patients. Am. J. Transplant. 2004; 4(9):1509-13. DOI: 10.1111/j.1600-6143.2004.00537.x 
21. Borges FSG. Caracterização da complexidade da terapêutica geriátrica [dissertation]. Covilhã: Universidade da Beira Interior; 2011.

22. Elliott R, O'callaghan C. Impact of Hospitalisation on the Complexity of Older Patients' Medication Regimens and Potential for Regimen Simplification. Journal of Pharmacy Practice and Research. 2011; 41(1):21-5. DOI: 10.1002/j.2055-2335.2011. tb00060.x

23. Santos APP, Silva DLM. Controle terapêutico no transplante renal: estratégia de promoção do uso racional de imunossupressores [monography]. Brasília: Universidade Católica de Brasília; 2010.

24. Kamila P, Smith SG, Patzer R, Wolf MS, Marina S. Medication Regimen Complexity in Kidney and Liver Transplant Recipients. Transplantation. 2014; 98(7). DOI: 10.1097/TP.0000000000000403.

Submetido em: 06/01/2017

Aceito em: 05/07/2017

Publicado em: 30/09/2017 